Konrad-Zuse-Zentrum für Informationstechnik Berlin

\title{
Online Call Admission in Optical Networks with Larger Wavelength Demands
}




\title{
ONLINE CALL ADMISSION IN OPTICAL NETWORKS WITH LARGER WAVELENGTH DEMANDS
}

\author{
SVEN O. KRUMKE AND DIANA POENSGEN ${ }^{1}$
}

\begin{abstract}
In the problem of Online Call Admission in Optical Networks, briefly called OCA, we are given a graph $G=(V, E)$ together with a set of wavelengths $W$ and a finite sequence $\sigma=r_{1}, r_{2}, \ldots$ of calls which arrive in an online fashion. Each call $r_{j}$ specifies a pair of nodes to be connected and an integral demand indicating the number of required lightpaths. A lightpath is a path in $G$ together with a wavelength $\lambda \in W$.

Upon arrival of a call, an online algorithm must decide immediately and irrevocably whether to accept or to reject the call without any knowledge of calls which appear later in the sequence. If the call is accepted, the algorithm must provide the requested number of lightpaths to connect the specified nodes. The essential restriction is the wavelength conflict constraint: each wavelength is available only once per edge, which implies that two lightpaths sharing an edge must have different wavelengths. Each accepted call contributes a benefit equal to its demand to the overall profit. The objective in OCA is to maximize the overall profit.

Competitive algorithms for OCA have been known for the special case where every call requests just a single lightpath. In this paper we present the first competitive online algorithms for the general case of larger demands.
\end{abstract}

\section{INTRODUCTION}

In current telecommunication networks, data is sent as optical signals of a chosen wavelength over glass fiber cables, using the wavelength division multiplexing (WDM) technique. At intermediate nodes the signals are converted back into electronic form, switched and then transformed back into optical form to send it over the ongoing fiber. This o-e-o-conversion limits the speed of the connections. In next generation's fully optical networks, optical signals are no longer converted back into electronic form at intermediate nodes but switched optically. This increases the speed of connections. It also changes the mathematical properties of the communication networks, because the wavelength on which the signal is sent stays the same on the whole path between start and end node.

A connection in a fully optical network can be modeled as a lightpath, that is, a path together with a wavelength. Since each wavelength is available only once per fiber, lightpaths used to establish connections must have different wavelengths if they use the same fiber at the same time. This crucial condition is called the wavelength conflict constraint.

\footnotetext{
${ }^{1}$ Konrad-Zuse-Zentrum für Informationstechnik Berlin, Department Optimization, Takustr. 7, D14195 Berlin-Dahlem, Germany. Email: \{krumke, poensgen\}@zib.de. Research supported by the German Science Foundation (DFG, grant Gr 883/10)

Key words and phrases. Call Admission, Routing and Wavelength Allocation, Optical Networks, Competitive Analysis, Colorability.
} 
1.1. Problem definition. An instance of the Online Call Admission Problem in Optical Networks (OCA) consists of an undirected graph $G=(V, E)$ together with a set of $\chi$ eligible wavelengths $W=\left\{\lambda_{1}, \ldots, \lambda_{\chi}\right\}$ and a finite request sequence $\sigma=r_{1}, r_{2}, \ldots, r_{n}$ of calls. Each of the wavelengths in $W$ is available once per edge. We will use the terms wavelength and color interchangeably in the sequel. A lightpath is a pair $(P, \lambda)$, where $P$ is a path in $G$ and $\lambda \in W$ is a wavelength.

A call $r_{j}=\left(s_{j}, t_{j}, b_{j}\right)$ specifies the nodes $s_{j} \in V$ and $t_{j} \in V$ to be connected as well as the required number $b_{j} \in \mathbb{N}$ of lightpaths, that is, its demand. Upon arrival of a new request $r_{j}$, an algorithm for OCA must decide whether to route or to reject $r_{j}$. If the call is accepted, it must be routed through the network without violating the wavelength conflict constraint. Once accepted, a call can not be preempted: the lightpaths used for the call can not be changed or removed anymore. Each accepted call $r_{j}$ contributes the corresponding demand $b_{j}$ to the total profit obtained by an algorithm. The overall goal of OCA is to maximize the total profit, that is, the total accepted demand.

An online algorithm for OCA must base its decision for call $r_{j}$ without knowledge of calls $r_{i}$ with $i>j$. A standard tool to measure the quality of an online algorithm ALG is competitive analysis, where one compares for each input sequence $\sigma$ the profit ALG $(\sigma)$ obtained by ALG to the optimal profit achievable on that sequence, denoted by $\operatorname{OPT}(\sigma)$.

Definition 1.1 (Competitive Deterministic Algorithm). A deterministic online algorithm ALG for OCA is c-competitive if for any request sequence $\sigma$ the inequality $\operatorname{ALG}(\sigma) \geq \frac{1}{c} \cdot \mathrm{OPT}(\sigma)$ holds.

A randomized online algorithm is a probability distribution over a set of deterministic online algorithms. The objective value produced by a randomized algorithm is therefore a random variable. In this paper we analyze the performance of randomized online algorithms against an oblivious adversary. An oblivious adversary knows the online algorithm and the distributions it uses, but does not see the realizations of the random choices made by the online algorithm and therefore has to generate a request sequence in advance. We refer to [BEY98] for details on the various adversary models.

Definition 1.2 (Competitive Randomized Algorithm). A randomized online algorithm RALG for OCA is defined to be c-competitive against an oblivious adversary if for any request sequence $\sigma$ the inequality $\mathbb{E}[\operatorname{RALG}(\sigma)] \geq \frac{1}{c} \cdot \mathrm{OPT}(\sigma)$ holds.

The competitive ratio of an algorithm is defined to be the infimum over all $c$ such that the algorithm is $c$-competitive.

1.2. Previous work. If the set of eligible wavelengths $W$ contains only a single wavelength, the problem of providing lightpaths reduces to the problem of finding edge disjoint paths in the given graph, which we will refer to as Edge Disjoint Path Allocation (EDPA). Competitive algorithms for EDPA are known for special graphs like lines, trees, and meshes. The currently best competitive ratios of randomized algorithms against an oblivious adversary for these topologies are $\lceil\log n\rceil$ for the line with $n$ nodes [AYFR94, $\mathrm{AAF}^{+}$96], $2 \log n$ for a tree with $n$ nodes [AYFR94, $\mathrm{AAF}^{+}$96, LMSPR98] and $\mathcal{O}(\log n)$ for the $n \times n$-mesh [KT95, LMSPR98].

So far, OCA with $\chi>1$ wavelengths has been investigated only for the special case in which each call requires one lightpath, i.e., $b_{j}=1$ for all $j$. Awerbuch et al. 
$\left(\left[\mathrm{AAF}^{+} 96\right]\right)$ developed the competitive algorithm FFC (First-Fit-Coloring), which is based on a "virtual" online algorithm for EDPA.

Theorem 1.3 ([AAF+96]). Let SLAVE be a c-competitive algorithm for EDPA. Then there is a $(c+1)$-competitive algorithm FFC for the special case of OCA where each call requires one lightpath.

Note that the competitive ratio of FFC does not depend on the number of eligible wavelengths in the network and differs from that of the subroutine used for EDPA only by an additive constant of 1 .

1.3. Our contribution. We present the first competitive algorithms for the general case of OCA in which the demand of a call may be greater than 1 . We assume, however, that no call asks for more than $\chi$ lightpaths. This assumption is reasonable since accepting a call of demand higher than $\chi$ (if at all possible) would plug up the network immediately. In particular, on trees our assumption means no restriction.

The first of our algorithms, Copy-Coloring (CC), is deterministic and works for OCA in general graphs. Our second algorithm, called First-fit-coloring-scaled (FFCS), is randomized, and for trees and the line achieves a competitive ratio which is exponentially better than CC's ratio. Table 1 gives an overview of our results together with the known lower bounds for EDPA from the literature.

\begin{tabular}{|l|l|l|l|}
\hline Topology & $\begin{array}{l}\text { competitive ratio } \\
\text { using generic } \\
c \text {-competitive } \\
\text { algorithm for EDPA }\end{array}$ & $\begin{array}{l}\text { competitive ratio } \\
\text { using best known } \\
\text { competitive } \\
\text { algorithm for EDPA }\end{array}$ & $\begin{array}{l}\text { known } \\
\text { lower bounds } \\
\text { for EDPA } \\
(\text { OCA with } \chi=1))\end{array}$ \\
\hline \hline $\begin{array}{l}\text { arbitrary network } \\
\text { with } n \text { nodes, } \\
\chi \text { wavelengths }\end{array}$ & $\begin{array}{l}c \cdot \chi \cdot d_{G} \\
(\text { Theorem } 2.1)\end{array}$ & $\begin{array}{l}\mathcal{O}(\chi \log n) \\
\text { on } n \times n \text { meshes }\end{array}$ & $\begin{array}{l}\text { deterministic: } n-1 \\
\text { randomized: } n^{1-\log _{4} 3} \\
{[\text { BFL96] }}\end{array}$ \\
\hline $\begin{array}{l}\text { tree with } n \text { nodes, } \\
\chi \text { wavelengths }\end{array}$ & $\begin{array}{l}12(c+1)(\lceil\log \chi\rceil+1) \\
(\text { Theorem 3.2) }\end{array}$ & $\begin{array}{l}(24 \log n+2) \cdot \\
(\lceil\log \chi\rceil+1)\end{array}$ & $\begin{array}{r}\text { deterministic: } n-1 \\
\text { randomized: }\left\lfloor\log \frac{n}{2}\right\rfloor \\
{\left[\text { AAF }^{+} 96\right]}\end{array}$ \\
\hline $\begin{array}{l}\text { line with } n \text { nodes, } \\
\chi \text { wavelengths }\end{array}$ & $\begin{array}{l}8(c+1)(\lceil\log \chi\rceil+1) \\
(\text { Theorem 3.7) }\end{array}$ & $\begin{array}{l}(8\lceil\log n\rceil+8) \cdot \\
(\lceil\log \chi\rceil+1)\end{array}$ & $\begin{array}{r}\text { deterministic: } n-1 \\
\text { randomized: }\left\lfloor\log \frac{n}{2}\right\rfloor \\
{\left[\text { AAF }^{+} 96\right]}\end{array}$ \\
\hline
\end{tabular}

TABLE 1. Results in Online Call Admission in Optical Networks.

\section{A Deterministic Algorithm for General Graphs}

Let the graph $G=(V, E)$ together with the set of eligible wavelengths $W=$ $\left\{\lambda_{1}, \ldots, \lambda_{\chi}\right\}$ be given in an arbitrary instance of OCA. Remember that the EDPA problem can be considered to be a special case of OCA in which there is only one eligible wavelength (and calls have demand 1). The deterministic online algorithm Copy Coloring CC uses an algorithm for EDPA as a subroutine. This algorithm, called SLAVE in the sequel, works on the instance of EDPA given by the graph $G$ and a single wavelength. 


\section{Algorithm Copy Coloring (cc)}

Let SLAVE be an online algorithm for EDPA. Upon arrival of a call $r_{j}=$ $\left(s_{j}, t_{j}, b_{j}\right)$ with demand $b_{j}$, hand the "sized down" call $\tilde{r}_{j}=\left(s_{j}, t_{j}, 1\right)$ to SLAVE.

If SLAVE rejects $\tilde{r}_{j}$, reject $r_{j}$. If SLAVE accepts $\tilde{r}_{j}$ and routes it on path $P$, then accept $r_{j}$ and route its demand using the lightpaths $\left(P, \lambda_{1}\right), \ldots,\left(P, \lambda_{b_{j}}\right)$.

It is easy to see that CC yields a valid solution. Recall that each call has demand at most $\chi$. If we view the graph $G$ together with its set of $\chi$ eligible colors as $\chi$ copies $G_{1}, \ldots, G_{\chi}$ of $G$, each in a different color, then SLAVE creates a feasible routing for the accepted calls from the modified sequence in $G_{1}$. In each remaining $G_{i}$, a subset of the paths routed in $G_{1}$ is established. Therefore, we have a feasible routing in each color, i.e., the wavelength conflict constraint is satisfied.

Theorem 2.1. Let $G$ be a graph with $\chi$ eligible wavelengths and maximum vertex degree $d_{G}$. If SLAVE is a c-competitive algorithm for EDPA, then CC is $\left(\chi \cdot d_{G} \cdot c\right)$ competitive on $G$.

Proof. By ALG A $_{k}$ we denote the algorithm ALG which has only the first $k$ colors of $W$ at its disposal (and can therefore only handle sequences of calls with demand at most $k$ ). In particular, $\mathrm{OPT}_{1}$ is the optimal offline algorithm for EDPA on the given graph $G$ and $\mathrm{OPT}_{\chi}$ is the optimal offline algorithm for OCA with $\chi$ eligible wavelengths. Let $\sigma^{(q)}$ be the sequence obtained from $\sigma$ by changing the demand of each call to $q$. Note that the maximum number of edge disjoint paths connecting any two nodes in $G$ is bounded by $d_{G}$.

Given a sequence $\sigma$ of calls, consider the maximum number of calls in $\sigma^{(1)}$ that can be routed simultaneously in one color (i.e., by edge disjoint paths). By definition this number equals $\mathrm{OPT}_{1}\left(\sigma^{(1)}\right)$. Since SLAVE is $c$-competitive for EDPA, we have $\operatorname{SLAVE}\left(\sigma^{(1)}\right) \geq \frac{1}{c} \mathrm{OPT}_{1}\left(\sigma^{(1)}\right)$.

Let $r_{j}$ be a call which is routed by the optimal offline algorithm OPT $\chi$ on lightpaths $\left(P, \lambda_{i_{1}}\right), \ldots,\left(P, \lambda_{i_{b_{j}}}\right)$. Each of the lightpaths will be referred to as a fragment of $r_{j}$. Let $\mathrm{OPT}_{\chi}(i, \sigma)$ denote the share of profit that OPT $=\mathrm{OPT}_{\chi}$ gains by call fragments routed in color $i$. Obviously this number is bounded from above by $d_{G} \cdot \mathrm{OPT}_{1}\left(\sigma^{(1)}\right)$. As a consequence, we have that

$$
\mathrm{OPT}_{\chi}(\sigma)=\sum_{i=1}^{\chi} \mathrm{OPT}(i, \sigma) \leq \chi \cdot d_{G} \cdot \mathrm{OPT}_{1}\left(\sigma^{(1)}\right) \leq \chi \cdot d_{G} \cdot c \cdot \operatorname{SLAVE}\left(\sigma^{(1)}\right),
$$

where the last inequality follows from the aforementioned competitiveness of SLAVE for EDPA. Clearly, CC makes as least as much profit on $\sigma$ as the SLAVE algorithm it uses on $\sigma^{(1)}$. Therefore,

$$
\operatorname{OPT}_{\chi}(\sigma) \leq \chi \cdot d_{G} \cdot c \cdot \operatorname{Cc}(\sigma),
$$

which shows the claim of the theorem.

At first glance, the competitive ratio of $\mathrm{CC}$ does not seem to be very good. However, the following theorem shows that without restrictions the bound achieved by CC is essentially the best which we can expect for deterministic algorithms. 
Theorem 2.2. On a line with $n$ nodes, no deterministic algorithm for OCA can be c-competitive with $c<\chi(n-1)$.

Proof. The worst case sequence is a straightforward generalization of the known lower bound construction from [BFL96] for EDPA on the line with $n$ nodes. Let the nodes be numbered by $v_{1}, v_{2}, \ldots, v_{n}$ from left to right. The adversary first issues a request $r_{1}=\left(v_{1}, v_{n}, 1\right)$. It is straightforward to see that any deterministic algorithm which achieves a finite competitive ratio must accept $r_{1}$. The adversary then presents the $n-1$ requests $\left(v_{1}, v_{2}, \chi\right),\left(v_{2}, v_{3}, \chi\right), \ldots,\left(v_{n-1}, v_{n}, \chi\right)$, none of which the deterministic online algorithm can accept.

\section{An Improved RANDOMized Algorithm FOR TREeS}

In this section we present the randomized algorithm First-Fit-Coloring-Scaled (FFCS) and analyze its performance on trees and the line. For these graph classes FFCS achieves an exponential improvement in the competitive ratio compared to the deterministic algorithm CC from the previous section.

We derive FFCS as a probability distribution over a set of $\lceil\log \chi\rceil+1$ deterministic algorithms which we denote by $\mathrm{FFCS}^{i}, i=0, \ldots,\lceil\log \chi\rceil$. Recall that $\sigma^{(q)}$ is the sequence obtained from sequence $\sigma$ by changing the demand of each call to $q$, and that $\mathrm{ALG}_{k}$ is the algorithm ALG working on a graph having $k$ wavelengths at its disposal. If the subscript is omitted, we always refer to the original problem in which we are given the graph $G$ together with $\chi$ eligible wavelengths.

\section{Algorithm First Fit Scaled (FFCS)}

Partition the set of possible calls into $\lceil\log \chi\rceil+1$ classes as follows: Class $K_{0}$ contains all calls with demand 1 . For $i=1, \ldots,\lceil\log \chi\rceil$, class $K_{i}$ contains those calls whose demand is in $\left(2^{i-1}, 2^{i}\right]$.

Choose $i \in\{0, \ldots,\lceil\log \chi\rceil\}$ uniformly at random and from this point on, use the deterministic algorithm FFCS ${ }^{i}$.

FFCS $^{i}$ : If call $r_{j}=\left(s_{j}, t_{j}, b_{j}\right)$ does not belong to class $K_{i}$, reject $r_{j}$. Otherwise size the demand of $r_{j}$ down to 1 and hand the modified call $\tilde{r}_{j}=\left(s_{j}, t_{j}, 1\right)$ over to $\mathrm{FFC}_{\left\lfloor\chi / 2^{i}\right\rfloor}$, that is the version of FFC which works on $G$ but has only $\left\lfloor\chi / 2^{i}\right\rfloor$ wavelengths $\left\{w_{1}, \ldots, w_{\left\lfloor\chi / 2^{i}\right\rfloor}\right\}$ at its disposal.

If FFC ${ }_{\left\lfloor\chi / 2^{i}\right\rfloor}$ rejects modified call $\tilde{r}_{j}$, then reject $r_{j}$. If FFC $\mathrm{F}_{\left\lfloor\chi / 2^{i}\right\rfloor}$ accepts the modified call $\tilde{r}_{j}$ and routes in on path $P$ in wavelength $w_{k}$, accept the original call $r_{j}$ and route it on the lightpaths $\left(P, \lambda_{(k-1) 2^{i}+1}\right),\left(P, \lambda_{(k-1) 2^{i}+2}\right), \ldots,\left(P, \lambda_{(k-1) 2^{i}+b_{j}}\right)$.

Proposition 3.1. FFCS produces a valid routing for the calls.

Proof. Let $i$ be the value of the random choice by FFCS. If a call $r_{j}=\left(s_{j}, t_{j}, b_{j}\right)$ is accepted by FFCS, it must belong the class class $K_{i}$, implying that $b_{j} \leq 2^{i}$, and the sized-down call $\tilde{r}_{j}=\left(s_{j}, t_{j}, 1\right)$ must be accepted by $\mathrm{FFC}_{\left\lfloor\chi / 2^{i}\right\rfloor}$.

The algorithm $\mathrm{FFC}_{\left\lfloor\chi / 2^{i}\right\rfloor}$ produces a valid routing in the graph $G$ for the set of accepted calls from $\sigma^{(1)}$. Since each wavelength $w_{k}$ of $\mathrm{FFC}_{\left\lfloor\chi / 2^{i}\right\rfloor}$ corresponds to a set $\lambda_{(k-1) \cdot 2^{i}+1}, \ldots, \lambda_{k \cdot 2^{i}}$ of $2^{i}$ wavelengths in the original graph, it follows that all accepted calls (which, as mentioned, have all demand at most $2^{i}$ ) can in fact be routed as specified without violating the wavelength conflict constraint. 
Theorem 3.2. Suppose that the following two conditions are satisfied:

(i) Each algorithm $\mathrm{FFC}_{\left\lfloor\chi / 2^{i}\right\rfloor}$ uses a c-competitive algorithm for the EDPA problem as a subroutine.

(ii) For any input sequence $\pi$ with the property that $\frac{b}{2}<b_{j} \leq b$ for all requests $r_{j}=\left(s_{j}, t_{j}, b_{j}\right) \in \pi$ the estimate

$$
\mathrm{OPT}_{\chi}(\pi) \leq c^{\prime} \cdot b \cdot \mathrm{OPT}_{\lfloor\chi / b\rfloor}\left(\pi^{(1)}\right)
$$

holds. Then, the randomized algorithm FFCS (with $\chi$ wavelengths at its disposal) achieves a competitive ratio of

$$
\left.2 c^{\prime}(c+1)(\lceil\log (\chi)\rceil+1)\right)
$$

Proof. Let $\sigma$ be an arbitrary call sequence. We have to show that the expected profit of FFCS satisfies

$$
\mathbb{E}[\operatorname{FFCS}(\sigma)] \geq \frac{1}{2 c^{\prime}(c+1)(\lceil\log (\chi)\rceil+1)} \cdot \operatorname{OPT}(\sigma) .
$$

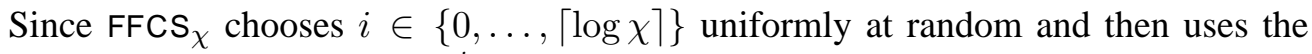
deterministic algorithm FFCS ${ }^{i}$ we can rewrite the left hand side of (1) as

$$
\mathbb{E}[\operatorname{FFCS}(\sigma)]=\frac{1}{\lceil\log (\chi)\rceil+1} \cdot \sum_{i=0}^{\lceil\log \chi\rceil} \operatorname{FFCS}^{i}(\sigma) .
$$

Recall that the deterministic algorithm FFCS ${ }^{i}$ rejects all calls which do not belong to class $K_{i}$. Thus, it only gains profit on calls from $K_{i}$, and we have that $\operatorname{FFCS}^{i}(\sigma)=$ $\operatorname{FFCS}^{i}\left(\left.\sigma\right|_{K_{i}}\right)$, where $\left.\sigma\right|_{K_{i}}$ is the subsequence of $\sigma$ which consists of calls belonging to $K_{i}$. Using this equality gives us

$$
\mathbb{E}[\operatorname{FFCS}(\sigma)]=\frac{1}{\lceil\log (\chi)\rceil+1} \cdot \sum_{i=0}^{\lceil\log \chi\rceil} \operatorname{FFCS}^{i}\left(\left.\sigma\right|_{K_{i}}\right) .
$$

Let $P_{i}^{*}(\sigma)$ denote that share of the total optimal profit which is gained with calls in $K_{i}$, that is,

$$
P_{i}^{*}(\sigma)=\sum_{\begin{array}{c}
\left.r \in \sigma\right|_{K_{i}} \\
r \text { is accepted by OPT } \\
\text { when given input } \sigma
\end{array}} b_{j} .
$$

Therefore, $\mathrm{OPT}_{\chi}(\sigma)=\sum_{i=0}^{\lceil\log \chi\rceil} P_{i}^{*}(\sigma)$. We now compare FFCs ${ }^{i}\left(\left.\sigma\right|_{K_{i}}\right)$ with $P_{i}^{*}(\sigma)$.

How big can the share of OPT's profit gained from $K_{i}$ be? $P_{i}^{*}(\sigma)$ gets largest if OPT uses all its resources for calls from $K_{i}$, which would be the optimal profit gained if only the sequence $\left.\sigma\right|_{K_{i}}$ was given. Therefore, $P_{i}^{*}(\sigma) \leq \mathrm{OPT}\left(\left.\sigma\right|_{K_{i}}\right)$, and this yields

$$
\operatorname{OPT}(\sigma)=\sum_{i=0}^{\lceil\log \chi\rceil} P_{i}^{*}(\sigma) \leq \sum_{i=0}^{\lceil\log \chi\rceil} \operatorname{OPT}\left(\left.\sigma\right|_{K_{i}}\right) .
$$

Hence, it suffices to upper bound the profit OPT $\left(\left.\sigma\right|_{K_{i}}\right)$ in terms of FFCS ${ }^{i}\left(\left.\sigma\right|_{K_{i}}\right)$. To this end we estimate the profit gained by the deterministic algorithm FFCS ${ }^{i}$ on the sequence $\left.\sigma\right|_{K_{i}}$. By construction, FFCS $^{i}$ accepts those calls whose modified version is accepted by $\mathrm{FFC}_{\left\lfloor\chi / 2^{i}\right\rfloor}$. As $\mathrm{FFC}_{\left\lfloor\chi / 2^{i}\right\rfloor}$ gets profit 1 for each accepted call (it was given calls whose demand was sized down to 1 ), the number of accepted calls equals 
FFC $_{\left\lfloor\chi / 2^{i}\right\rfloor}\left(\left(\left.\sigma\right|_{K_{i}}\right)^{(1)}\right)$. Since FFCS ${ }^{i}$ gets profit $b_{j} \geq 2^{i-1}$ for each accepted call, we obtain that

$$
\operatorname{FFCS}^{i}\left(\left.\sigma\right|_{K_{i}}\right) \geq 2^{i-1} \cdot \operatorname{FFC}_{\left\lfloor\frac{\chi}{2^{i}}\right\rfloor}\left(\left(\left.\sigma\right|_{K_{i}}\right)^{(1)}\right) .
$$

We now apply Theorem 1.3 about the competitiveness of FFC to FFC $\left\lfloor\frac{x}{2^{i}}\right\rfloor$. This results in

$$
\operatorname{FFCS}^{i}\left(\left.\sigma\right|_{K_{i}}\right) \geq 2^{i-1} \cdot \frac{1}{c+1} \cdot \mathrm{OPT}_{\left\lfloor\frac{\chi}{2^{i}}\right\rfloor}\left(\left(\left.\sigma\right|_{K_{i}}\right)^{(1)}\right) .
$$

Observe that all the demands in $\left.\sigma\right|_{K_{i}}$ are within a factor of two. Hence, we can use assumption (ii) with $\pi=\left.\sigma\right|_{K_{i}}$ and $b=2^{i}$ to obtain:

$$
\mathrm{OPT}_{\left\lfloor\frac{\chi}{2^{i}}\right\rfloor}\left(\left(\left.\sigma\right|_{K_{i}}\right)^{(1)}\right) \geq \frac{1}{c^{\prime} 2^{i}} \cdot \mathrm{OPT}_{\chi}\left(\left.\sigma\right|_{K_{i}}\right) .
$$

Plugging (6) into (5) and using this result in (3) gives

$$
\mathbb{E}[\operatorname{FFCS}(\sigma)] \geq \frac{1}{\lceil\log (\chi)\rceil+1} \sum_{i=0}^{\lceil\log \chi\rceil} \frac{1}{2 c^{\prime}(c+1)} \cdot \operatorname{OPT}_{\chi}\left(\left.\sigma\right|_{K_{i}}\right)
$$

The claim of the theorem now follows from (4).

Theorem 3.2 bounds the competitive ratio of FFCS in terms of (i) the competitive ratio $c$ of a virtual online algorithm for EDPA, and (ii) the ratio $c^{\prime}$ between the optimal offline profit $\mathrm{OPT}_{\chi}(\pi)$ and $b$ times the optimal offline profit on a scaled sequence $\pi^{(1)}$ with fewer wavelengths $\lfloor\chi / b\rfloor$.

In the sequel we address the existence of the second ratio $c^{\prime}$ for the case of trees and, as a special case, for the line.

3.1. Call Coloring on Trees. Note that on trees, the problem of finding a feasible routing for a given set of calls reduces to the problem of path coloring, since each call uniquely determines the path to be used. Therefore, we will also speak of call coloring or path coloring.

Lemma 3.3. Let $G$ be a tree. Let $\pi$ be a call sequence such that the demand $b_{j}$ of each call in $\pi$ satisfies $\frac{b}{2}<b_{j} \leq b$. Then

$$
\mathrm{OPT}_{\chi}(\pi) \leq 6 \cdot b \cdot \mathrm{OPT}_{\left\lfloor\frac{\chi}{b}\right\rfloor}\left(\pi^{(1)}\right)
$$

Proof. We prove the claim in three steps. We first consider the following special case:

(a) $b_{j}=b$ for all $j$,

(b) $b$ divides $\chi$.

For this special case we show that

$$
\mathrm{OPT}_{\chi}(\pi) \leq \frac{3}{2} \cdot b \cdot \mathrm{OPT}_{\left\lfloor\frac{\chi}{b}\right\rfloor}\left(\pi^{(1)}\right) .
$$

We then show that we can drop the two assumptions one by one, losing factor 2 in each of the two steps.

Assume that conditions (a) and (b) hold. Let $S$ be the set of calls from sequence $\pi$ by which the optimal profit on the left hand side in (8) is achieved. Then OPT $\chi(\pi)=$ $|S| \cdot b$, since by assumption (a), the demand of each call equals $b$. If we were able to show that the calls in $S^{(1)}$, i.e., the paths corresponding to the calls in $S^{(1)}$, can be 
colored with $\frac{\chi}{b}$ colors, this would imply that the set $S^{(1)} \subset \pi^{(1)}$ could be accepted and routed feasibly by any algorithm which has $\frac{\chi}{b}$ colors at its disposal, yielding profit $|S|=\left|S^{(1)}\right|$. Since the optimal offline algorithm can do only better, we would obtain OPT $_{\frac{\chi}{b}}\left(\pi^{(1)}\right) \geq|S|=\frac{1}{b} \cdot$ OPT $_{\chi}(\pi)$.

Unfortunately, it is in general not possible to color all the calls in $S^{(1)}$ by $\frac{\chi}{b}$ colors. This is illustrated by Figure 1. It shows a tree $T$ of depth one and a set of 13 calls (i.e.paths) on $T$. It is possible to assign $b=2$ different colors to each of the given paths such that two intersecting paths have disjoint color sets and only $\chi=6$ colors are used overall. It is however impossible to assign 1 color to each of the paths using only $3=\chi / b$ colors such that intersecting paths have different colors.

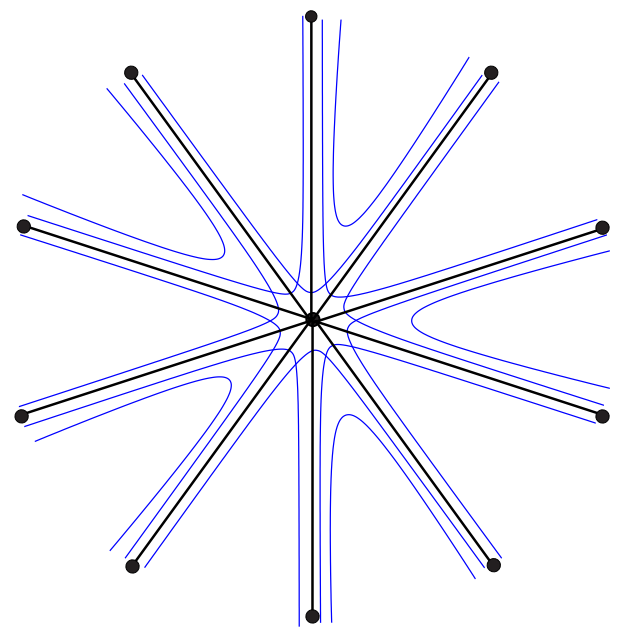

FIGURE 1. An example in which $\chi / b$ colors do not suffice to color all given paths $(\chi=6, b=2)$.

However, we will show that at least two third of the calls in $S^{(1)}$ can be colored using $\chi / b$ colors. Define the maximum (unweighted) load of a set of paths in a graph to be the maximum number of paths which have an edge in common.

Theorem 3.4 ([RU94]). There is a feasible routing of requests of maximum load $L$ per link of undirected trees using no more than $\frac{3}{2} L$ wavelengths.

We seek to apply Theorem 3.4. We know that there is a feasible routing for the calls in $S$, that is, each call in $S$ is assigned $b$ lightpaths (the uniquely determined path together with $b$ different colors), using at most $\chi$ different colors in total. On each edge, the maximum load of the whole set of lightpaths established to route $S$ is hence bounded from above by $\chi$. Therefore, the maximum load of paths (calls) in $S^{(1)}$ is bounded from above by $\frac{\chi}{b}$, as each call in $S^{(1)}$ corresponds to one path whereas a call in $S$ corresponds to $b$ lightpaths.

Applying Theorem 3.4 yields that there is a coloring of the calls in $S^{(1)}$ using $\frac{3}{2} \cdot \frac{\chi}{b}$ colors. If we consider those $\frac{\chi}{b}$ among the $\frac{3}{2} \cdot \frac{\chi}{b}$ colors from the coloring which accommodate the most calls, a simple averaging argument gives us that they accommodate a set $M^{(1)} \subset S^{(1)}$ of calls of cardinality at least $\frac{2}{3} \cdot\left|S^{(1)}\right|$. The optimal algorithm on 
$\chi / b$ colors might even accept a larger set of calls from $\pi^{1}$, so since $|S|=\left|S^{(1)}\right|$ and $b$ divides $\chi$, we can conclude that

$$
\mathrm{OPT}_{\left\lfloor\frac{\chi}{b}\right\rfloor}\left(\pi^{(1)}\right)=\mathrm{OPT}_{\frac{\chi}{b}}\left(\pi^{(1)}\right) \geq\left|M^{(1)}\right| \geq \frac{2}{3} \cdot|S|=\frac{2}{3 b} \cdot \mathrm{OPT}_{\chi}(\pi)
$$

which is exactly what we claimed in (8).

Now assume that only condition (b) holds, that is, $b$ divides $\chi$, but the demand $b_{j}$ of a call may take any integral value between $\frac{b}{2}$ and $b$, i.e., $\frac{b}{2}<b_{j} \leq b$. Consider the routing defined by $\mathrm{OPT}_{\chi}(\pi)$. Since $b-b_{j}<b_{j}$, we can accommodate $b-b_{j}$ additional lightpaths for each of the accepted calls if we provide $\chi$ additional colors. We thus get a valid solution for the sequence $\pi^{(b)}$ in the graph with $2 \chi$ wavelengths. We can conclude that $\mathrm{OPT}_{\chi}(\pi) \leq \mathrm{OPT}_{2 \chi}\left(\pi^{(b)}\right)$. As $b$ divides $\chi$, it also divides $2 \chi$, and since the demand of each call in $\pi^{b}$ equals $b$, we can apply inequality (8). We obtain

$\mathrm{OPT}_{\chi}(\pi) \leq \mathrm{OPT}_{2 \chi}\left(\pi^{b}\right) \stackrel{(8)}{\leq} \frac{3}{2} \cdot b \cdot \mathrm{OPT}_{\frac{2 \chi}{b}}\left(\pi^{(1)}\right) \leq \frac{3}{2} \cdot b \cdot 2 \cdot \mathrm{OPT}_{\frac{\chi}{b}}\left(\pi^{(1)}\right)=3 \cdot b \cdot \mathrm{OPT}_{\frac{\chi}{b}}\left(\pi^{(1)}\right)$,

where the last inequality holds as $\mathrm{OPT}_{\chi / b}$ can accept at least those calls from $\pi^{(1)}$ which were accepted by $\mathrm{OPT}_{2 \chi / b}$ and routed in the "fuller" half of the $\frac{2 \chi}{b}$ colors used.

We finally show that we can drop condition (b) using the part we just proved for the second inequality in the following chain:

$$
\mathrm{OPT}_{\chi}(\pi) \leq \mathrm{OPT}_{2 \cdot\left\lfloor\frac{\chi}{b}\right\rfloor \cdot b}(\pi) \leq 3 \cdot b \cdot \mathrm{OPT}_{2 \cdot\left\lfloor\frac{\chi}{b}\right\rfloor}\left(\pi^{(1)}\right) \leq 2 \cdot 3 \cdot b \cdot \mathrm{OPT}_{\left\lfloor\frac{\chi}{b}\right\rfloor}\left(\pi^{(1)}\right) .
$$

The first inequality simply holds because $\chi \leq 2 \cdot\left\lfloor\frac{\chi}{b}\right\rfloor \cdot b$, the last by the same reasoning as before: the "fuller" half of the $2 \cdot\left\lfloor\frac{\chi}{b}\right\rfloor$ colors accommodates at least half of the calls.

This completes the proof of the lemma.

Corollary 3.5. If there is a c-competitive algorithm for EDPA on trees, then FFCS achieves a competitive ratio of $12(c+1)(\lceil\log \chi\rceil+1)$ for routing in $\chi$ wavelengths.

Using one of the $2 \log n$-competitive algorithms for EDPA on trees with $n$ vertices from [AYFR94, $\left.\mathrm{AAF}^{+} 96, \mathrm{LMSPR} 98\right]$, this results in a $(24 \log n+2)(\lceil\log \chi\rceil+1)$ competitive algorithm for OCA on the same graph class.

3.2. Call Coloring on Paths. In case that the underlying graph is a simple path, a slightly better result than the one in Lemma 3.3 can be achieved.

Lemma 3.6. Let $G$ be a path. Let $\pi$ be a call sequence such that the demand $b_{j}$ of each call in $\pi$ satisfies $\frac{b}{2}<b_{j} \leq b$. Then

$$
\mathrm{OPT}_{\chi}(\pi) \leq 4 \cdot b \cdot \mathrm{OPT}_{\left\lfloor\frac{\chi}{b}\right\rfloor}\left(\pi^{(1)}\right)
$$

Proof. As before, we first consider the case that all demands satisfy $b_{j}=b$ for all $j$ and that $b$ divides $\chi$. For this case, we show that

$$
\mathrm{OPT}_{\chi}(\pi)=b \cdot \mathrm{OPT}_{\left\lfloor\frac{\chi}{b}\right\rfloor}\left(\pi^{(1)}\right) \text {. }
$$

As shown in the proof of Lemma 3.3, dropping the two assumptions above costs us a factor of 2 each. This yields the desired result.

It remains to prove (9). It is straightforward to see that $\mathrm{OPT}_{\chi}(\pi) \geq b \cdot \mathrm{OPT}_{\left\lfloor\frac{\chi}{b}\right\rfloor}\left(^{(1)}\right)$. We will now show that the inequality also holds the other way around, namely that $\mathrm{OPT}_{\chi}(\pi) \leq b \cdot \mathrm{OPT}_{\left\lfloor\frac{\chi}{b}\right\rfloor}\left(\pi^{(1)}\right)$. 
Let $A \subseteq \pi$ be the set of calls accepted by $\mathrm{OPT}_{\chi}$ on input $\pi$. Consider the following auxiliary graph $H$ with vertex set corresponding to the calls in $A$ : we insert one (interval-) vertex for each of the $b$ call fragments of each accepted call. Two vertices in $H$ are adjacent if the corresponding call fragments share an edge. Clearly, $H$ is an interval graph with maximum clique size $\omega(H) \leq \chi$.

We consider the subgraph $H^{\prime}$ obtained from $H$ by removing for each call $r \in A$ all but one of the $b$ vertices representing its call fragments and their adjacent edges. Hence, the vertices in $H^{\prime}$ correspond to a subset of the calls in $\pi^{(1)}$. Clearly, $\omega\left(H^{\prime}\right)=$ $\omega(H) / b \leq \chi / b$. Since interval graphs are perfect, we can color the vertices in $H^{\prime}$ using at most $\omega\left(H^{\prime}\right)=\chi / b$ colors. Thus, the coloring of the calls in $A$ translates into a solution for the $\pi^{(1)}$-instance in which as many calls (of profit 1 ) are accepted by $\mathrm{OPT}_{\frac{\chi}{b}}$ on $\pi^{(1)}$ as calls (with profit $b$ ) are accepted by $\mathrm{OPT}_{\chi}$ on $\pi$.

Corollary 3.7. If there is a c-competitive algorithm for EDPA on the line, then FFCS achieves a competitive ratio of $8(c+1)(\lceil\log \chi\rceil+1)$ for routing in $\chi$ wavelengths.

\section{REFERENCES}

$\left[\mathrm{AAF}^{+} 96\right]$ B. Awerbuch, Y. Azar, A. Fiat, S. Leonardi, and A. Rosén, On-line competitive algorithms for call admission in optical networks, Proceedings of the 4th Annual European Symposium on Algorithms, vol. 1136, 1996, pp. 431-444.

[AYFR94] B. Awerbuch, Y.Bartal, A. Fiat, and A. Rosén, Competitive, non-preemptive call control, Proceedings of the 5th Annual ACM-SIAM Symposium on Discrete Algorithms, 1994, pp. 312-320.

[BEY98] A. Borodin and R. El-Yaniv, Online computation and competitive analysis, Cambridge University Press, 1998.

[BFL96] Y. Bartal, A. Fiat, and S. Leonardi, Lower bounds for on-line graph problems with applications to on-line curcuit and optimal routing, Proceedings of the 28th Annual ACM Symposium on the Theory of Computing, 1996, pp. 531-540.

[KT95] J. Kleinberg and E. Tardos, Disjoint paths in densely embedded graphs, Proceedings of the 36th Annual IEEE Symposium on the Foundations of Computer Science, 1995, pp. 531540.

[LMSPR98] S. Leonardi, A. Marchetti-Spaccamela, A. Presciutti, and A. Rosèn, On-line randomized call-control revisited, Proceedings of the 9th Annual ACM-SIAM Symposium on Discrete Algorithms, 1998, pp. 323-332.

[RU94] P. Raghavan and E. Upfal, Efficient routing in all-optical networks, Proceedings of the 26th Annual ACM Symposium on the Theory of Computing, 1994, pp. 134-143.

Sven O. Krumke, Konrad-Zuse-Zentrum für Informationstechnik Berlin, DepartMEnT Optimization, TAKustr. 7, D-14195 Berlin-Dahlem, Germany.

E-mail address: krumke@ zib.de

URL: http: //www.zib.de/krumke

Diana Poensgen, Konrad-Zuse-Zentrum FÜr InFormationstechniK Berlin, DePartMENT Optimization, TAKustr. 7, D-14195 Berlin-DAhlem, Germany.

E-mail address: poensgen@zib.de 